\title{
It is Time to Uncover the Mysterious veil of Atrioventricular Node Pacing
}

\author{
Cheng-ming $\mathrm{Ma}^{1}$, Yong-mei $\mathrm{Cha}^{2}$, yingxue Dong ${ }^{1}$, Lianjun $\mathrm{Gao}^{3}$, and Yunlong Xia ${ }^{3}$ \\ ${ }^{1}$ First Affiliated Hospital of Dalian Medical University \\ ${ }^{2}$ Mayo Clinic \\ ${ }^{3}$ The First Affiliated Hospital of Dalian Medical University
}

March 13, 2021

\begin{abstract}
Wenckebach phenomenon is a well-known electrophysiological character of the atrioventricular node (AVN). AVN-pacing during permanent pacemaker implantation is rare. We herein report the first case of Wenckebach phenomenon in a heart failure patient with chronic atrial fibrillation for more than 30 years during the His-bundle pacing (HBP) procedure. The patient's symptoms improved significantly. AVN-pacing is available, and HBP is helpful for cardiac remodeling and clinical outcomes.
\end{abstract}

\section{Hosted file}

Entire manuscript.pdf available at https://authorea.com/users/401348/articles/513465-it-istime-to-uncover-the-mysterious-veil-of-atrioventricular-node-pacing 